\title{
Pesquisa
}

\section{CONSIDERAÇÕES SOBRE A SAÚDE MENTAL DO HOMEM}

\author{
Benjamin Silwamba Kahozi (1) \\ Antônio Cláudio Carlos de Freitas Filho(2) \\ Marcio de Paiva Bezerra Carrah (3) \\ Plínio Henrique Fernandes Leandro (4) \\ Bárbara Maria de Macêdo (5)
}

\begin{abstract}
Resumo
O objetivo do presente estudo foi conhecer aspectos relacionados à saúde geral do homem, na região do Crajubar. Considerou-se uma análise comparativa das variáveis (saúde geral e síndrome de burnout), de 102 homens entre 20 e 58 anos de idade Eles responderam a um instrumento, contendo o Questionário de Saúde Geral (QSG-12) e o Inventário de Burnout de Maslach (MBI). Pode-se avaliar que, em relação à amostra objeto do presente estudo, houve um maior comprometimento da saúde emocional, na faixa etária que vai de 21 a 30 anos de idade. Confia-se que os objetivos deste estudo tenham sido alcançados parcialmente, uma vez que outras variáveis ainda estão sendo analisadas. Resta, entretanto, averiguar o que ocorre com as demais variáveis, a exemplo de renda, escolaridade e religiosidade dos participantes. Além disso, reconhecendo o caráter multidimensional do estresse laboral, seria importante incluir medidas complementares, sobretudo aquelas que permitam cobrir uma dimensão afetiva maior, relacionada a satisfação com a vida, avaliando em que fatores os homens podem diferir em termos deste construto. Estes são certamente desafios que demandarão atenção em pesquisas futuras. É possível que estudos que controlem variáveis, aqui ainda não consideradas, apontem para uma outra direção, em termos de estresse laboral. Dessa forma, torna-se mister levar à cabo mais estudos dessa natureza. Estes, em adição ao presente trabalho, conformarão uma base teórica, para entendimento de quais aspectos poderiam ajudar na promoção de uma vida mais saudável e com maior bem-estar, para os homens.
\end{abstract}

Palavras - chaves : Qualidade de vida; Gênero e Saúde; Burnout; Estresse

\section{Introdução}

A saúde mental pode ser definida como sendo, um nível de qualidade de vida cognitiva ou emocional, ou a ausência de uma doença mental. Mas, segundo a Organização 
Mundial de Saúde, não existe uma definição "oficial" de saúde mental. Diferenças culturais, julgamentos subjetivos, e teorias relacionadas concorrentes, afetam o modo como a "saúde mental" é definida (WHO, 2001).

Estabelecer uma inter-relação entre a saúde mental e o trabalho sempre foi, e ainda é, um desafio necessário e complexo para pesquisadores e profissionais das áreas da Saúde e do Trabalho. Tornou uma questão prioritária para a área da saúde, principalmente no Brasil. País capitalista, onde se vive uma grande desigualdade e, se constata que: quem tem mais, paga mais e recebe mais, enquanto quem tem menos, recebe o proporcional a isso. Essas condições prejudicam não somente pessoas da classe menos favorecida ou com poder aquisitivo baixo, mas também os que se encontram no topo da escala econômica como médicos e empresários, levando assim a uma má qualidade de saúde mental e consequentemente sujeitando essas pessoas ao adoecimento.

Atualmente percebe-se que, no processo saúde - doença, fatores biológicos e genéticos não são as únicas causas de patologias. Levamos também em conta como agravantes, fatores ambientais, sócio - culturais, pessoais, organizacionais e aspectos circunstanciais. Sabemos também que, apesar do crescimento econômicos e das mudanças nas formas de produção, de instrumentos de trabalho e da velocidade das comunicações em virtude de uma maior demanda, o trabalhador, na tentativa de adaptar-se, tem chegado a limites de exigência física e mental, principalmente o homem, que menos se cuida e mais adoece em relação às mulheres (GOMES, 2007).

Uma mente preocupada não tem um bom rendimento, menos ainda uma pessoa doente (HELOANI e CAPITÃO, 2003). Prova disso são os constantes absenteísmos e diminuição da jornada de trabalho, devido ao adoecimento (GOMES, 2007).

Diante desta realidade, nos propusemos a conhecer aspectos relacionados a saúde geral do homem na região do Crajubar. Para isso foi necessário caracterizar a amostra sócioculturalmente, conhecer a situação da amostra quanto a síndrome de burnout e comparar a situação de saúde geral da amostra, relacionando-a com algumas variáveis tal como escolaridade e religião. Acreditamos que o conhecimento desses aspectos provocará uma reflexão sobre essa realidade, de forma a impulsionar programas de prevenção a saúde do homem. Alguns autores acreditam que os problemas provocados ou agravados pelo trabalho, não afetam somente o homem, mas toda uma sociedade (PRISZKULNIK, 2009; MORUFUSE, N.T., ABRANCHES, S.S. e NAPOLEÃO, A.A., 2005). 


\section{Método}

A presente pesquisa propôs-se a um estudo transversal, quantitativo, correlacional, do tipo ex post-facto. Considerou-se uma análise comparativa das variáveis (saúde geral e síndrome de burnout), no grupo de sujeitos considerados.

A amostra foi formada por 102 homens da região do CRAJUBAR, que compreende os municípios de Crato, Juazeiro e Barbalha, na região sul do Ceará. A escolha do tema deveuse ao fato da baixa busca nos serviços de saúde pelos homens, em comparação com as mulheres.

Os participantes responderam a um instrumento, contendo as seguintes partes:

a) Questionário de Saúde Geral (QSG-12). Este instrumento corresponde a uma versão abreviada do Questionário de Saúde Geral de Goldberg (1972), adaptado para o Brasil por Pasquali e cols. (1994). Como sua abreviação indica, a versão que é utilizada neste estudo se compõe de 12 itens (por exemplo: Você tem se sentido pouco feliz e deprimido?; Você tem perdido a confiança em si mesmo?). Cada item respondido em termos do quanto a pessoa tem experimentado os sintomas descritos, sendo suas respostas dadas em uma escala de quatro pontos. No caso dos itens que negam a saúde mental (por exemplo: Suas preocupações lhe têm feito perder muito sono?; Tem se sentido pouco feliz e deprimido?), as alternativas de resposta variam de 1 (Absolutamente, não) a 4 (Muito mais que de costume); no caso dos itens afirmativos (por exemplo: Tem se sentido capaz de tomar decisões?; Tem podido concentrar-se bem no que faz?), as respostas foram de 1 (Mais que de costume) a 4 (Muito menos que de costume). Os itens negativos foram invertidos, de modo que a maior pontuação total nesta medida indica melhor nível de saúde mental. Como antes indicado, os estudos realizados no Brasil, inclusive sem definir uma estrutura fatorial única, concordam sobre a existência de um fator geral, com Alfa de Cronbach variando de 0,80 (SARRIERA, SCHWARCZ e CÂMARA, 1996) a 0,88 (BORGES e ARGOLO, 2002).

b) Inventário de Burnout de Maslach (MBI). Para avaliar a Síndrome de Burnout foi utilizado o MBI - Maslach Burnout Inventory, que tem a tradução validada para a língua portuguesa por Benevides-Pereira (2001). O inventário é auto-aplicado e avalia como o sujeito vivencia seu trabalho, de acordo com as três dimensões estabelecidas pelo Modelo Teórico de Maslach: Exaustão Emocional (9 itens), Realização Pessoal no Trabalho (RP) (8 itens) e Despersonalização (5 itens). Totaliza, portanto, 22 itens que indicam a freqüência das respostas com uma escala de 6 pontos variando de 1 a 7 . Neste estudo, adotou-se o sistema de pontuação de 1 a 5, utilizado por Tamayo (1997) na adaptação brasileira do instrumento, 
pois foi verificado que os sujeitos apresentavam dificuldade em responder muitos itens dos instrumentos, devido à especificidade dos critérios da escala original. Utilizamos, portanto, 1 para "nunca", 2 para "algumas vezes ao ano", 3 para "algumas vezes ao mês", 4 para indicar "algumas vezes na semana" e 5 para "diariamente". A consistência interna das três dimensões do inventário é satisfatória, pois apresenta um alfa de Cronbach que vai de 0,71 até 0,90 (MASLACH e JACKSON,1981).

c) Informações Sócio-Demográficas: Foi proposto um conjunto de perguntas visando caracterizar a amostra, a exemplo de idade, sexo, religião, escolaridade, ocupação, renda, dentre outras.

Os próprios pesquisadores foram instruídos em relação à uniformidade do procedimento necessário para aplicar o instrumento. Estes, visitaram diferentes bairros de Crato, Juazeiro do Norte e Barbalha, selecionando residências e locais públicos (por exemplo, praças e mercados) para a coleta dos dados. As pessoas foram solicitadas a responder voluntariamente a um questionário e, foram também informadas que o objetivo da pesquisa era de natureza científica, evitando que fosse confundida com propósitos políticos ou econômicos. Todos responderam ao instrumento individualmente. O tempo médio para responder aos questionários foi de 15 minutos. Procurou-se entrevistar pessoas do sexo masculino de idades variadas.

O Projeto foi encaminhado ao Comitê de Ética indicado pela Universidade Federal do Ceará, para autorização do trabalho de campo.

Os sujeitos foram devidamente esclarecidos do propósito do estudo, de acordo com a Resolução 196/96 do Conselho Nacional de Saúde, que trata das diretrizes de e normas regulamentadoras de pesquisas envolvendo seres humanos.

\section{Resultados}

\section{Caracterização do grupo investigado}

Compuseram o grupo da pesquisa, 102 homens, com idades variando entre 20 e 58 anos, de três municípios da região do Cariri cearense, no período de julho de 2010 . No intuito de melhor compreender os resultados obtidos, inicialmente, tratamos de apresentar informações que permitam uma caracterização desses sujeitos. 
A tabela 1 apresenta, a seguir, a distribuição dos sujeitos da amostra, em relação a sua faixa etária.

Tabela 1 - Distribuição dos sujeitos segundo a faixa etária. 2010.

\begin{tabular}{c|c|c}
\hline FAIXA ETÁRIA & $\mathrm{N}$ & $\%$ \\
\hline Até 20 anos & 1 & 1,0 \\
\hline 21 a 30 anos & 49 & 48,0 \\
\hline 31 a 40 anos & 23 & 22,6 \\
\hline 41 a 50 anos & 21 & 20,6 \\
\hline Maior que 51 anos & 8 & 7,8 \\
\hline TOTAL & 102 & 100,0 \\
\hline
\end{tabular}

As idades dos participantes variaram entre 20 e 58 anos, sendo 49 sujeitos (48,0\%) com idades entre 21 a 30 anos.

Com relação à situação conjugal dos sujeitos que participaram da pesquisa, estes se declararam: Casados/Conviventes $(45,1 \%)$ ou Solteiros $(46,1 \%)$. Uma minoria $(8,8 \%)$ afirmou ser Separado/Divorciado.

Quanto à religião, em sua maioria, os sujeitos estudados são católicos $(63,7 \%)$, sendo ainda $14,7 \%$ protestantes; $1,0 \%$ espíritas, $3,9 \%$ se autodenominaram com outra religião e, $16,7 \%$ se reconheceram agnósticos.

Para a caracterização socioeconômica, em termos de classe social a que pertence, foi sugerida aos sujeitos uma escala de 1 (classe baixa) a 10 (classe alta), cujos resultados, encontram-se na tabela seguinte.

Tabela 2- Distribuição dos sujeitos de acordo com a classe social informada. 2010.

\begin{tabular}{l|c|c}
\hline \multicolumn{1}{c|}{ CLASSE SOCIAL } & $\mathrm{N}$ & $\%$ \\
\hline Baixa $(1,2$ e 3$)$ & 18 & 17,6 \\
\hline Média $(4,5,6$ e 7$)$ & 80 & 78,5 \\
\hline Alta $(8,9$ e 10$)$ & 4 & 3,9 \\
\hline TOTAL & 102 & 100,0 \\
\hline
\end{tabular}

A classe mais predominante foi a média, com 78,5\% dos participantes, seguida da baixa com $17,6 \%$.

Quanto à escolaridade dos participantes, pudemos observar que a amostra se apresenta bem diversificada, a saber: 3,9\% não completou o ensino fundamental; 5,9\% 
completou o ensino fundamental; $3,9 \%$ possui o ensino médio incompleto; $34,3 \%$ possui o ensino médio completo; $25,5 \%$ possui o curso superior incompleto e $26,5 \%$ completou o ensino superior. A escolarização dos sujeitos é fator importante para a saúde.

Adicionalmente, procuramos conhecer a situação laboral dos entrevistados, tendo em vista o que nos informa Maturano (1997), que os aspectos socioeconômicos interferem no desenvolvimento humano e nas condições de saúde.

Observou-se que, 95,1\% dos participantes estava empregado à época da pesquisa. $1,0 \%$ estava desempregado e 1,0\% encontrava-se afastado temporariamente. Apenas 2,0\% respondeu estar em outra situação. A Ocupação dos sujeitos, neste sentido, é um fator importante para uma boa performance, em termos de cuidados de saúde. O fator econômico é também um poderoso catalisador da existência de cuidados higiênicos e de acompanhamento da saúde em todas as faixas de renda.

A tabela a seguir, apresenta a função exercida atualmente pelos sujeitos.

Tabela 3 - Distribuição dos sujeitos de acordo com a função exercida atualmente em termos laborais. 2010.

\begin{tabular}{l|c|c}
\multicolumn{1}{c|}{ FUNÇÃO ATUAL } & N & $\%$ \\
\hline $\begin{array}{l}\text { Desempregado } \\
\begin{array}{l}\text { Técnico de nível superior (advogado, } \\
\text { contador, médico, enfermeiro, auditor, } \\
\text { educador físico, professor) }\end{array}\end{array}$ & 10 & 1,0 \\
\hline $\begin{array}{l}\text { Técnico de nível médio } \\
\text { Área comercial (atendente, gerente, } \\
\text { balconista, operadores de micro, } \\
\text { vendedores, agente de atendimento, etc) }\end{array}$ & 28 & 9,8 \\
\hline $\begin{array}{l}\text { Serviços gerais (limpeza e conservação, } \\
\text { porteiro, vigilante) }\end{array}$ & 37 & 36,3 \\
\hline $\begin{array}{l}\text { Autônomos (tatuador, eletricista, } \\
\text { fotógrafo, motoboy, pedreiro, chaveiro, } \\
\text { empresário, etc) }\end{array}$ & 13 & 6,8 \\
\hline Outra & & 12,7 \\
\hline TOTAL & 6 & \\
\hline
\end{tabular}

Quanto a distribuição dos sujeitos de acordo com a função exercida atualmente, percebemos que a maioria dos entrevistados atuavam na área comercial (36,3\%) à época da pesquisa, logo depois se encontram os que atuavam como técnico de nível médio (27,5\%). Somente $12,7 \%$ eram autônomos. A maioria dos participantes não trabalhava em regime de plantão $(81,4 \%)$ e apenas $18,6 \%$ não atuavam sob este regime laboral. 
Com relação à Renda mensal, observe-se a tabela a seguir:

Tabela 4 - Distribuição dos sujeitos de acordo com a renda mensal. 2010.

\begin{tabular}{l|c|c}
\hline \multicolumn{1}{c|}{ RENDA MENSAL } & $\mathrm{N}$ & $\%$ \\
\hline Menos de 1 salário mínimo (SM)* & 16 & 15,8 \\
\hline De 1 a 3 Salários mínimos & 58 & 56,8 \\
\hline De 4 a 6 Salários mínimos & 15 & 14,7 \\
\hline De 7 a 9 Salários mínimos & 8 & 7,8 \\
\hline De 10 a 12 Salários mínimos & 3 & 2,9 \\
\hline Mais que 12 Salários mínimos & 2 & 2,0 \\
\hline TOTAL & 102 & 100,0 \\
\hline
\end{tabular}

*Salário mínimo vigente em julho/2010, R\$ 510,00.

Percebemos que $15,8 \%$ dos participantes recebem um salário menor que o mínimo, exigido pela legislação em vigor. Outros $12,7 \%$ recebem mais de seis salários mínimos, enquanto que a maior concentração dos sujeitos ocupou a faixa entre um a três salários mínimos $(56,8 \%)$.

$\mathrm{Na}$ dimensão econômica, os dados nos levam a crer que existe muita insatisfação laboral em pelo menos $50 \%$ dos casos, já que os sujeitos responderam que, em caso de possibilidade, desejariam mudar de profissão.

A tabela abaixo nos mostra a situação dos sujeitos da amostra, com relação a Saúde Geral:

Tabela 5 - Distribuição dos sujeitos de acordo com a Saúde Geral medida pelo QSG-12. 2010 .

\begin{tabular}{c|c}
\hline FAIXA DE IDADE & $\begin{array}{c}\text { PONTUAÇÃO } \\
\text { NO QSG-12 }\end{array}$ \\
\hline Até $20^{\mathrm{a}}$ & 17 \\
& $\mathrm{Sd}=1,00$ \\
\hline 21 a $30^{\mathrm{a}}$ & 22,31 \\
& $\mathrm{Sd}=5,459$ \\
\hline 31 a $40^{\mathrm{a}}$ & 20,22 \\
& $\mathrm{Sd}=3,954$ \\
\hline 41 a $50^{\mathrm{a}}$ & 20,13 \\
& $\mathrm{Sd}=4,220$ \\
\hline Mais que $50^{\mathrm{a}}$ & 20,13 \\
& $\mathrm{Sd}=4,220$ \\
\hline Considerando a média de & 21,60 \\
todas as idades & $\mathrm{Sd}=4,853$ \\
\hline
\end{tabular}


Observe-se que, de uma maneira Geral, os homens pontuaram na média, sem grandes comprometimentos quanto a sua saúde psicológica $(21,60 . \mathrm{Sd}=4,853)$. No caso das pessoas com idades entre 21 a 30 anos, estas se mostraram um tanto mais comprometidas em termos de ansiedade e possibilidade de comportamento depressivo (22,31. $\mathrm{Sd}=5,459)$. Enquanto os de mais de 50 anos se encontram na média com 20,13 $(\mathrm{Sd}=4,220)$ os de até 20 anos flutuam $(17,0 . \mathrm{Sd}=1,00)$.

Com relação à Síndrome de Burnout, a tabela abaixo nos mostra a situação dos sujeitos da amostra:

Tabela 6 - Distribuição dos sujeitos de acordo com a Síndrome de Burnout. 2010.

\begin{tabular}{l|c|c|c}
\hline $\begin{array}{c}\text { FAIXA DE } \\
\text { IDADE }\end{array}$ & $\begin{array}{c}\text { PONTUAÇÃO } \\
\text { EE }\end{array}$ & $\begin{array}{c}\text { PONTUAÇÃO } \\
\text { DP }\end{array}$ & $\begin{array}{c}\text { PONTUAÇÃO } \\
\text { EPT }\end{array}$ \\
\hline Até $20^{\mathrm{a}}$ & 9 & 10 & 36 \\
\hline $21 \mathrm{a} 30^{\mathrm{a}}$ & 12 & 16,47 & 31,20 \\
\hline $31 \mathrm{a} 40^{\mathrm{a}}$ & 11,78 & 15,61 & 31,91 \\
\hline $41 \mathrm{a} 50^{\mathrm{a}}$ & 8,80 & 13,88 & 32,25 \\
\hline Mais que $50^{\mathrm{a}}$ & 8,88 & 13,88 & 33,25 \\
\hline $\begin{array}{l}\text { Considerando a } \\
\text { média de todas as } \\
\text { idades }\end{array}$ & 11,28 & 15,72 & 31,73 \\
\hline
\end{tabular}

Com relação à síndrome de burnout, as pontuações médias em relação a todas as idades, não demonstraram comprometimento no tocante a Exaustão Emocional (EE). Quanto a Despersonalização (DP), os índices foram considerados altos, de acordo com a escala de Maslach, sendo maior na faixa de 21 a 30 anos de idade $(16,47 . \mathrm{Sd}=5,025)$. Com relação à Realização pessoal no trabalho (EPT), os índices se apresentaram bons, havendo uma tendência a crescer à medida que a idade avança.

\section{Conclusões}

Neste estudo, procurou-se inicialmente conhecer as correlações da pontuação total da medida de saúde geral, com respeito ao conjunto de variáveis consideradas, tanto demográficas como do contexto laboral. Para conhecer a consistência de tais correlações, os cálculos levaram em conta, principalmente a idade dos homens participantes. 
Pode-se avaliar que, em relação à amostra, houve um maior comprometimento de saúde emocional na faixa etária que vai de 21 a 30 anos de idade.

Feitas essas ponderações, confia-se que os objetivos deste estudo tenham sido alcançados parcialmente, uma vez que outras variáveis ainda estão sendo analisadas. Foram reunidas evidências empíricas que explicam similaridades entre os correlatos demográficos e laborais da saúde geral e da síndrome de burnout em uma amostra eminentemente masculina.

Resta, entretanto, averiguar o que ocorre com as demais variáveis, a exemplo de renda, escolaridade e religiosidade dos participantes.

Além disso, reconhecendo o caráter multidimensional do estresse laboral, seria importante incluir medidas complementares, sobretudo aquelas que permitam cobrir uma dimensão afetiva maior, relacionada a satisfação com a vida, avaliando em que fatores os homens podem diferir em termos deste construto. Estes são certamente desafios que demandarão atenção em pesquisas futuras.

\section{Referências}

BENEVIDES-PEREIRA, A. M. T. A saúde mental de profissionais de saúde mental. Maringá: EDUEM, 2001.

GOMES, Romeu; DO NASCIMENTO, Elaine Ferreira; DE ARAÚJO, Fábio Carvalho. Por que os homens buscam menos os serviços de saúde do que as mulheres? As explicações de homens com baixa escolaridade e homens com ensino superior. Cad. Saúde Pública vol.23 no.3 Rio de Janeiro Mar. 2007.

HELOANI, José Roberto; CAPITÃO, Cláudio Garcia. Saúde mental e psicologia do trabalho. São Paulo Perspec. vol.17 no.2 São Paulo Apr./June 2003.

MASLACH, C. \& JACKSON, S. E. The measurement of experienced burnout. Journal of Occupational Behavior, 2, 99-113, 1981.

MASLACH, C. \& JACKSON, S. Maslach Burnout Inventory Manual. Palo Alto, CA: Consulting Psichologist Press, 1986.

MUROFUSE, Neide Tiemi; ABRANCHES, Sueli Soldati; NAPOLEÃO, Anamaria Alves. Reflexões sobre estresse e Burnout e a relação com a enfermagem. Rev. Latino-Am. Enfermagem vol.13 no.2 Ribeirão Preto Mar./Apr. 2005.

PASQUALI, L., GOUVEIA, V. V., ANDRIOLA, W. B. MIRANDA, F. J. e RAMOS, A. L. M. Questionário de Saúde Geral de Goldberg (QSG): Adaptação brasileira. Psicologia: Teoria e Pesquisa, 10 (3), 421-438, 1994.

PRISZKULNIK, Léia. Prevenção: saúde mental e psicanálise. An 7 Col. LEPSI IP/FE-USP 2009.

SARRIERA, J. C., SCHWARCZ, C. e CÂMARA, S. G. (1996). Bem-estar psicológico: análise fatorial da Escala de Goldberg (QSG-12) numa amostra de jovens. Psicologia: Reflexão e Crítica, 9, 293-306. 
TAMAYO, M. Relação entre a Síndrome de Burnout e os valores organizacionais no pessoal de enfermagem de dois hospitais públicos. Dissertação de Mestrado,

Universidade de Brasília, Brasília, 1997

WHO. Disponível em <http://www.who.int/whr/2001/chapter1/en/index.html >. Acesso em 15/11/2001.

Sobre os autores:

(1) Benjamin Silwamba Kahozi é Acadêmico de Medicina da Universidade Federal do Ceará - UFC. E-mail: kahozi_t9@yahoo.fr

(2) Antônio Cláudio Carlos de Freitas Filho é Acadêmico de Medicina da Universidade Federal do Ceará - UFC. E-mail: claudioffilho@ hotmail.com

(3) Marcio de Paiva Bezerra Carrah é Acadêmico de Medicina da Universidade Federal do Ceará UFC. E-mail: marciocarrah@yahoo.com.br

(4) Plínio Henrique Fernandes Leandro é de Medicina da Universidade Federal do Ceará - UFC. E-mail: pliniofernandes01@ hotmail.com

(5) Bárbara Maria de Macêdo é Médica pela Faculdade de Medicina de Juazeiro do Norte - CE. E-mail: babymacedo19@yahoo.com.br

\section{Como citar este artigo (Formato ISO):}

KAHOZI, B.S.; FREITAS FILHO, A.C.C.; CARRAH, M.P.B.; LEANDRO, P.H.F.; MACÊDO, B.M. Considerações sobre a saúde mental do homem. Id on Line Revista de Psicologia, Novembro/ 2010, vol.1, no.12, p.80-89. ISSN 1981-1189. 